\title{
Continuous-variable entanglement and two-mode squeezing in a single-atom Raman laser
}

\author{
Eyob A. Sete ${ }^{1, *}$ and C. H. Raymond $\mathrm{Ooi}^{2}$ \\ ${ }^{1}$ Institute for Quantum Science and Engineering and Department of Physics and Astronomy, Texas A\&M University, \\ College Station, Texas 77843-4242, USA \\ ${ }^{2}$ Department of Physics, University of Malaya, Kuala Lumpur 50603, Malaysia
}

(Received 19 March 2012; published 18 June 2012)

\begin{abstract}
The quantum statistical properties of light emitted by a two-photon double Raman laser is investigated. Using the master equation derived in the good-cavity limit, we study the squeezing and entanglement properties of the cavity field. It turns out that the cavity radiation exhibits two-mode squeezing and entanglement in the transient as well as steady state regime for realizable parameters. We establish a connection between two-mode squeezing and entanglement that gives insight into the physical origin of these quantum features. We also discuss the interplay between the laser detuning and amplitude in modifying the properties of the cavity field.
\end{abstract}

DOI: 10.1103/PhysRevA.85.063819

PACS number(s): 42.50.Ct, 03.67.Bg, 42.50.Nn

\section{INTRODUCTION}

Squeezing and entanglement are quantum-mechanical properties of radiation which do not have a classical analog. The former is generated as a result of certain nonlinear processes. Typical examples include optical parametric oscillators [1-3], which involve second-order nonlinearities expressed via the susceptibility $\chi^{(2)}$, and four-wave mixing expressed via the third-order nonlinearity $\chi^{(3)}[2,4]$. Squeezing has been experimentally demonstrated in the aforementioned systems and many other quantum optical systems [5]. It has potential applications in the detection of weak signals like a gravitational wave [6], optical communications, and measurements [7]. Recently, two-mode-squeezing sources have been linked to the generation of a macroscopic entangled state of radiation. On the other hand, quantum entanglement was shown to be a key resource for quantum information processing such as quantum teleportation [8,9] and quantum cryptography [10].

In recent years, quantum entanglement in continuous variables has received much attention due to its accessibility by experiment [11]. Among others, two-photon lasers are promising candidates for the generation of two-mode continuous-variable entanglement. Zubairy and coworkers proposed an entanglement amplifier with a large number of photons via correlated-emission lasers (CELs) [12]. This work, however, is limited to the transient regime and hence the entanglement is short lived. Subsequent studies have shown steady-state entanglement in CELs [13-15] and double Raman lasers [16] in the presence of losses and atomic relaxations. In addition, entanglement based on quantum-beat lasers has been put forward by a number of authors [17-19]. More recently, single-atom lasers have also been considered as a continuous source of entangled light with a large number of photons $[16,20]$, which later was demonstrated experimentally in a single-mode cavity setup [21].

In this work, we study quantum-statistical properties of radiation emitted by a single-atom double Raman laser in the presence of atomic relaxations and cavity losses. The transient

\footnotetext{
${ }^{*}$ Current address: Department of Electrical Engineering, University of California Riverside, Riverside, CA 92521, USA; eyobas@physics.tamu.edu
}

entanglement properties of this model have been investigated $[16,20]$. Here, we mainly focus on the squeezing properties and steady state entanglement behavior of the cavity radiation. Note that, while the generation of entanglement in two-photon lasers crucially relies on the atomic coherence induced either by initially injecting atoms in a coherent superposition of atomic levels or by coupling these levels by strong laser field, the type of entanglement created varies based on the configuration of the gain medium. As a consequence of this variation, the criteria that can be used to detect entanglement are quite different as well. For example, if the gain medium constitutes three-level atoms in a cascade configuration, as in a CEL, one would expect a two-photon entangled state of the form $\alpha\left|1_{1} 1_{2}\right\rangle+\beta\left|0_{1} 0_{2}\right\rangle$ with $\alpha$ and $\beta$ being complex numbers, which can only be detected by a certain class of entanglement criteria [22-26]. However, for the quantum-beat laser, where the gain medium is an ensemble of a V-type three-level atoms, the generated entanglement has the form $\alpha\left|1_{1} 0_{2}\right\rangle+\beta\left|0_{1} 1_{2}\right\rangle$ due to the quantum inference between the two possible pathways that lead to the lower level. In general, the entanglement criteria for two-photon entangled states fail to detect single-photon entangled states and vise versa $[22,27,28]$. Given that our system involves two-photon processes and exhibits non Gaussian photon statistics, the entanglement criteria proposed in [22-26] are sufficient to detect the entanglement in our scheme. Our results show that the cavity field can be in an entangled state both in the transient and steady state regimes for realizable parameters. The cavitymode field also exhibits two-mode squeezing for the same set of parameters. A simple formula that relates the two-mode squeezing and entanglement conditions is established. This connection sheds light on the physical origin of these quantum phenomena.

The paper is organized as follows: In Sec. II, we present the model and derive the master equation for the cavity radiation in the good-cavity limit, which is valid for an atom-field coupling constant that is small compared to other system parameters. In Sec. III, we present our main results. We show that the cavity mode exhibits transient and steady state squeezing for a wide range of system parameters. Moreover, using Hillery-Zubairy [22], logarithmic negativity [23-25], and Duan et al. [26], sufficient inseparability conditions for non-Gaussian two-mode states, we show that the cavity field 


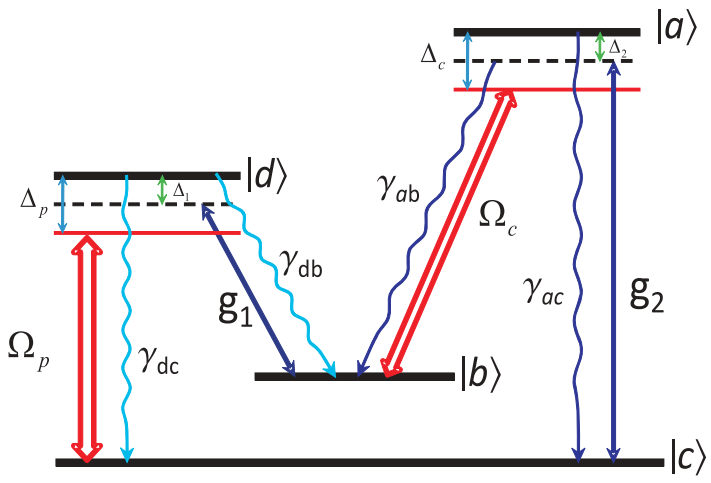

FIG. 1. (Color online) Energy level diagram for double Raman atom. The pump laser of Rabi frequency $\Omega_{p}$ and control laser of Rabi frequency $\Omega_{c}$ are off-resonantly coupled to levels $|c\rangle$ and $|d\rangle$, and $|a\rangle$ and $|b\rangle$, respectively. The quantized Stokes and anti-Stokes photons are off resonantly coupled to transitions $|d\rangle \leftrightarrow|b\rangle$ and $|a\rangle \leftrightarrow|c\rangle$ via the couplings $\mathrm{g}_{1}$ and $\mathrm{g}_{2}$, respectively. $\gamma_{i j}(i, j=a, b, c, d)$ are spontaneous emission rates.

of the double Raman laser can be in an entangled state for realizable system parameters. We finally conclude by presenting our main results in Sec. IV.

\section{MASTER EQUATION}

Our model consists of a single double Raman atom in a double resonant cavity. A pump laser with Rabi frequency $\Omega_{p}$ couples levels $|c\rangle$ and $|d\rangle$ while a control laser of Rabi frequency $\Omega_{c}$ couples levels $|b\rangle$ and $|a\rangle$ (see Fig. 1). Note that the pump and control lasers are used to create two-photon coherence which is responsible for demonstration of two-mode quadrature squeezing and entanglement, as discussed in Sec. III. Besides, the atom off-resonantly interacts with the quantized cavity modes: Stokes and anti-Stokes. The interaction Hamiltonian which describes the interaction of the external lasers and cavity modes with the atom is given, in the dipole and rotating wave approximations, by

$$
\begin{aligned}
H= & -\hbar\left(\Omega_{p}|d\rangle\left\langle c\left|e^{-i \Delta_{p} t}+\Omega_{c}\right| a\right\rangle\langle b| e^{-i \Delta_{c} t}\right. \\
& \left.+\mathrm{g}_{1}|d\rangle\left\langle b\left|\hat{a}_{1} e^{-i \Delta_{1} t}+\mathrm{g}_{2}\right| a\right\rangle\langle c| \hat{a}_{2} e^{-i \Delta_{2} t}\right)+ \text { H.c. },
\end{aligned}
$$

where $\Delta_{p}=v_{p}-\omega_{d b}, \Delta_{c}=v_{c}-\omega_{a c}$, with $v_{p}\left(v_{c}\right)$ being the pump (control) laser frequency and $\omega_{d b}\left(\omega_{a c}\right)$ being the atomic transition frequency for the $|d\rangle \rightarrow|b\rangle(|a\rangle \rightarrow|c\rangle)$ transition. $\mathrm{g}_{1}\left(\mathrm{~g}_{2}\right)$ is the Stokes (anti-Stokes) atom coupling constant, and $\Delta_{1}=v_{s}-\omega_{d b}, \Delta_{2}=v_{a}-\omega_{a c}$ with $v_{s}\left(v_{a}\right)$ being the Stokes (anti-Stokes) mode frequency.

The master equation of the cavity field derived, following a standard laser theory methods [29], in the good-cavity limit $(\kappa \ll \gamma, \mathrm{g})$ and linear approximation scheme reads

$$
\begin{aligned}
\frac{d \hat{\rho}}{d t}= & {\left[\mathcal{L}_{1}\left(\hat{a}_{1} \hat{\rho} \hat{a}_{1}^{\dagger}-\hat{\rho} \hat{a}_{1}^{\dagger} \hat{a}_{1}\right)+\mathcal{G}_{1}\left(\hat{a}_{1}^{\dagger} \hat{\rho} \hat{a}_{1}-\hat{a}_{1} \hat{a}_{1}^{\dagger} \hat{\rho}\right)\right.} \\
& +\mathcal{L}_{2}\left(\hat{a_{2}} \hat{\rho} \hat{a}_{2}^{\dagger}-\hat{\rho} a_{2}^{\dagger} \hat{a}_{2}\right)+\mathcal{G}_{2}\left(\hat{a}_{2}^{\dagger} \hat{\rho} \hat{a}_{2}-\hat{a}_{2} \hat{a}_{2}^{\dagger} \hat{\rho}\right) \\
& \left.+e^{-i \varphi_{t}}\left(\mathcal{Q}_{1} \hat{a}_{2} \rho \hat{a}_{1}+\mathcal{Q}_{2} \hat{a}_{1} \hat{\rho} \hat{a}_{2}-\mathcal{Q}_{3} \hat{\rho} \hat{a}_{1} \hat{a}_{2}-\mathcal{Q}_{4} \hat{a}_{1} \hat{a}_{2} \hat{\rho}\right)\right] \\
& + \text { H.c., }
\end{aligned}
$$

where $\kappa$ is the cavity decay rate and $\gamma$ is the spontaneous emission rate assumed to be the same for all levels. $\mathcal{L}_{j}$, and $\mathcal{G}_{j}$ are loss and gain coefficients, whereas $\mathcal{Q}_{j}$ are phase-sensitive cross-coupling coefficients that are due to atomic coherence between levels $|b\rangle$ and $|c\rangle$. It is worthwhile mentioning here that the presence of two-photon annihilation $\hat{a}_{1} \hat{a}_{2}$ and creation $\hat{a}_{1}^{\dagger} \hat{a}_{2}^{\dagger}$ processes is a signature that the two-mode light would exhibit squeezing and entanglement. $\phi_{t}=\varphi_{p}+\varphi_{c}-\varphi_{1}-\varphi_{2}$ is an effective phase for the Raman process, and $\mathcal{Q}_{1}+\mathcal{Q}_{2}=$ $\mathcal{Q}_{3}+\mathcal{Q}_{4}$ [16]. Explicit expressions for $\mathcal{L}_{j}, \mathcal{G}_{j}$, and $\mathcal{Q}_{j}$ are given in the appendix.

\section{QUANTUM STATISTICAL PROPERTIES}

Using the solutions of the coupled equations for averages of the cavity field operators, we study the two-mode squeezing and entanglement using criteria that involve up to second-order correlations.

\section{A. Two-mode squeezing}

We seek to study the quadrature squeezing of the cavity radiation. To this end, we introduce quadrature operators for the two-mode cavity field as

$$
\begin{aligned}
& \hat{c}_{+}=\frac{1}{\sqrt{2}} \sum_{j=1}^{2}\left(\hat{a}_{j}^{\dagger}+\hat{a}_{j}\right), \\
& \hat{c}_{-}=\frac{i}{\sqrt{2}} \sum_{j=1}^{2}\left(\hat{a}_{j}^{\dagger}-\hat{a}_{j}\right) .
\end{aligned}
$$

Using these definitions the quadrature variances can be put in the form

$$
\begin{aligned}
& \Delta c_{ \pm}^{2} \\
& =1+\left\langle\hat{a}_{1}^{\dagger}, \hat{a}_{1}\right\rangle+\left\langle\hat{a}_{2}^{\dagger}, \hat{a}_{2}\right\rangle \\
& +\left[\left\langle\hat{a}_{1}, \hat{a}_{2}^{\dagger}\right\rangle \mp\left(\left\langle\hat{a}_{1}, \hat{a}_{2}\right\rangle+\frac{1}{2}\left\langle\hat{a}_{1}, \hat{a}_{1}\right\rangle+\frac{1}{2}\left\langle\hat{a}_{2}, \hat{a}_{2}\right\rangle\right)+\operatorname{adj} .\right],
\end{aligned}
$$

where we have used the notation $\langle a, b\rangle=\langle a b\rangle-\langle a\rangle\langle b\rangle$. The quadrature operators, which can be measured using homodyne experiment [30], satisfy the commutation relation $\left[\hat{c}_{+}, \hat{c}_{-}\right]=$ $2 i$. Thus the two-mode light is said to in a squeezed state if either $\Delta c_{-}^{2}<1$ or $\Delta c_{+}^{2}<1$ with $\Delta c_{+}^{2}=0$ or $\Delta c_{-}^{2}=0$ being perfect squeezing and 1 no squeezing. In the steady state the variances reduce to

$$
\Delta c_{ \pm}^{2}=1+\left\langle\hat{n}_{1}\right\rangle+\left\langle\hat{n}_{2}\right\rangle \pm 2 \operatorname{Re}\left[\left\langle\hat{a}_{1} \hat{a}_{2}\right\rangle\right]
$$

Here, $\hat{n}_{1}, \hat{n}_{2}$ are photon number operators for the cavity modes. It is easy to see from Eq. (6) that, for the squeezing to occur in the $\hat{c}_{+}$quadrature, that means the noise is reduced below the coherent or vacuum level, and the inequality $2 \operatorname{Re}\left(\left\langle\hat{a}_{1} \hat{a}_{2}\right\rangle\right)<$ $-\left(\left\langle\hat{n}_{1}\right\rangle+\left\langle\hat{n}_{2}\right\rangle\right)$ should be satisfied. However, for the squeezing to occur in the $\hat{c}_{-}$quadrature, $2 \operatorname{Re}\left(\left\langle\hat{a}_{1} \hat{a}_{2}\right\rangle\right)>\left(\left\langle\hat{n}_{1}\right\rangle+\left\langle\hat{n}_{2}\right\rangle\right)$. Note that the form of quadrature variance (6) is also valid when the cavity modes are initially in a vacuum state. This is because terms that appear in Eq. (6), $\left\langle\hat{a}_{1}\right\rangle,\left\langle\hat{a}_{2}\right\rangle$, and $\left\langle\hat{a}_{1}^{2}\right\rangle,\left\langle\hat{a}_{2}^{2}\right\rangle$, $\left\langle\hat{a}_{1} \hat{a}_{2}^{\dagger}\right\rangle$ are zero at all times for this particular initial condition as per the equations shown in the appendix. 


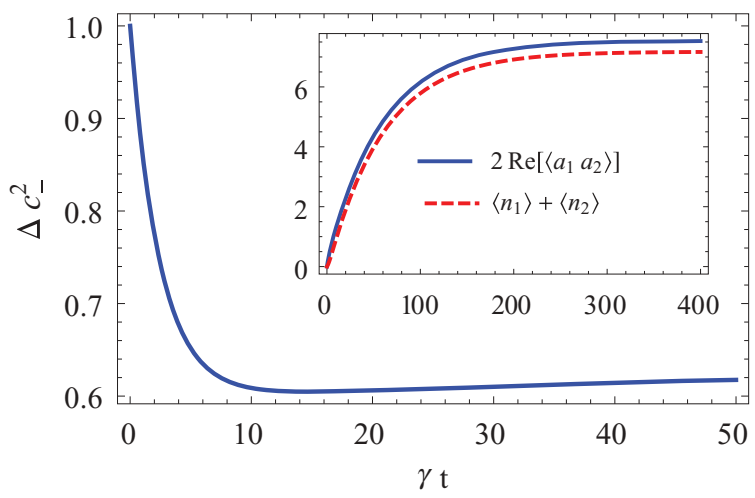

FIG. 2. (Color online) Temporal behavior of two-mode quadrature squeezing for $\Delta_{p}=\Delta_{c}=\Delta_{1}=\Delta_{2}=0$ and when the cavity modes are initially in a vacuum state. Here we have used $\gamma_{b c}=0, \gamma_{a c}=\gamma_{d c}=\gamma_{a d}=\gamma_{a b}=\gamma_{d b}=\gamma, \quad \Omega_{c}=\gamma, \Omega_{p}=$ $5 \gamma, \kappa_{1}=0.1 \gamma, \kappa_{2}=0.14 \gamma, \phi_{t}=\pi / 2, \mathrm{~g}_{1}=\mathrm{g}_{2}=\gamma$ and initial conditions $\rho_{a a}^{(0)}=\rho_{b b}^{(0)}=\rho_{a b}^{(0)}=0.5, \rho_{c d}^{(0)}=\rho_{d d}^{(0)}=\rho_{c c}^{(0)}=0$. The inset shows the time evolution of the intermode correlation and the total mean photon number.

\section{B. Transient squeezing}

Here we explore how squeezing of the cavity field evolves in time. We would like to mention that the exact analytical solutions are rather lengthy; we thus present our numerical results. First, we consider the temporal behavior of squeezing for the case of resonant Raman scheme $\left(\Delta_{p}=\Delta_{c}=0\right)$ and when the cavity modes are resonant with the Stokes and anti-Stokes transitions $\left(\Delta_{1}=\Delta_{2}=0\right)$. We also assume that the cavity modes are initially in a vacuum state. In Fig. 2, we plot the variance of the squeezed quadrature $\Delta c_{-}^{2}$ versus dimensionless time $\gamma t$ when the atom is initially in a coherent superposition of levels $|a\rangle$ and $|b\rangle$. This figure shows that the intracavity two-mode light is in a squeezed state with the reduction of noise nearly $40 \%$ below the vacuum level. The inset shows that the cross correlations are stronger than the total mean photon numbers, indicating squeezing of the cavity field noise. It is interesting to see that the total mean photon number gradually grows in time and attains its steady state, where the squeezing is stronger. Thus our scheme generates bright squeezed light in the long-time limit.

We next address the effect of the coherent pumping lasers on the degree of squeezing. Keeping the Rabi frequency of the control laser constant and varying the pump laser Rabi frequency, we show that the amount of squeezing of the two-mode intracavity light decreases when one increases the strength of the pump field [see Fig. 3(a)]. This can be understood by noting that the correlation between the Stokes and anti-Stokes photon strongly relies on the delay between the emission of the two photons. Note that, once the atom is excited to level $|a\rangle$ it may decay to level $|c\rangle$ via spontaneous emission or oscillates back and forth between levels $|a\rangle$ and $|b\rangle$, depending on the strength of the control field $\left(\Omega_{c}\right)$. For Rabi frequency of the control laser $\Omega_{c}$ less than or equal to the spontaneous emission rate $\gamma$, the atom predominantly decays to level $|c\rangle$. Once the atom is in level $|c\rangle$ the pump laser promotes the atom to level $|d\rangle$, which ensures subsequent emission of a Stokes photon. When the pump laser is strong enough to make Rabi oscillations, the likelihood of emission of the Stokes photon becomes smaller and hence the delay between the two photons becomes longer, which results in weaker correlation or squeezing. In essence, a very strong pump laser decreases the intermode correlation and hence the degree of squeezing. Note that the photon correlation also corresponds to the squeezing since both measure nonclassicality. Thus, a very strong pump laser decreases the intermode correlation and hence the degree of squeezing.

So far we have only considered resonance conditions in all cases $\left(\Delta_{p}=\Delta_{c}=\Delta_{1}=\Delta_{2}=0\right)$. It appears that the detuning can influence the degree of squeezing of the cavity radiation. In the following we assume the cavity modes are at resonance with the atom $\left(\Delta_{1}=\Delta_{2}=0\right)$ and the control laser is at resonance with the $|a\rangle$ to $|b\rangle$ transition $\left(\Delta_{c}=0\right)$. For instance, as illustrated in Fig. 3(b), the degree of squeezing decreases profoundly when detuning jumps from $\Delta_{p}=0$ to $\Delta_{p}=20 \gamma$. The underlying physics can be explained by noting that the intermode correlation crucially relies on the delay between the emission of the Stokes and anti-Stokes photons. This delay depends by how much the pump laser is detuned with respect to the $|c\rangle \rightarrow|d\rangle$ transition. The higher the detuning, the lesser the probability that the atom would be excited in short time, which in turn implies the longer delay between emission of the two photons and hence a weaker intermode correlations or squeezing. In effect, one should tune the pump laser close to resonance to obtain maximum squeezing. In order to clearly see this effect we next consider the long-time behavior.

\section{Steady state squeezing}

As mentioned in the above discussion, the variance of the squeezed quadrature operator becomes constant in the
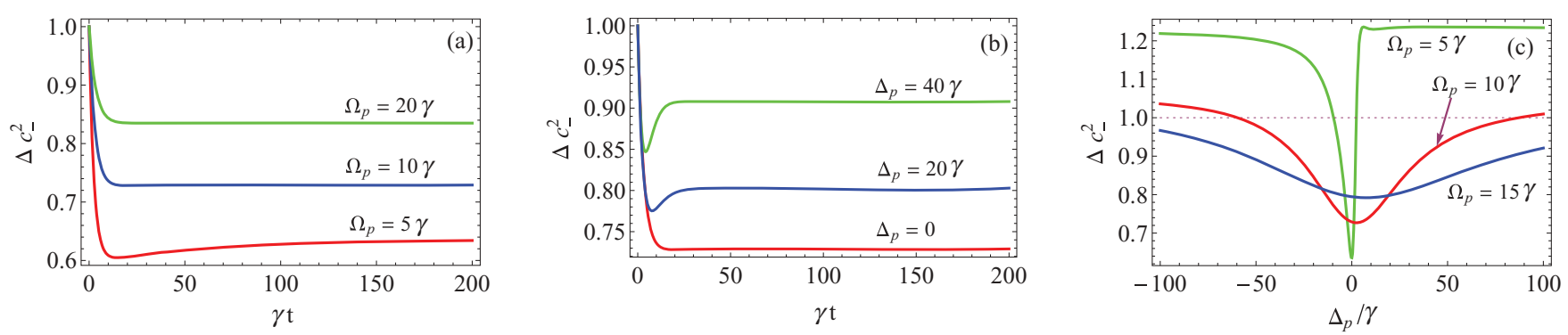

FIG. 3. (Color online) Temporal behavior of two-mode quadrature squeezing when cavity modes are initially in a vacuum state (a) for $\Delta_{p}=0$ and for various values of $\Omega_{p}$ (b) for $\Omega_{p}=10 \gamma$ and various values of $\Delta_{p}$. (c) Steady state squeezing versus $\Delta_{p} / \gamma$ for various values of $\Omega_{p}$. All other parameters are the same as Fig. 2 . 

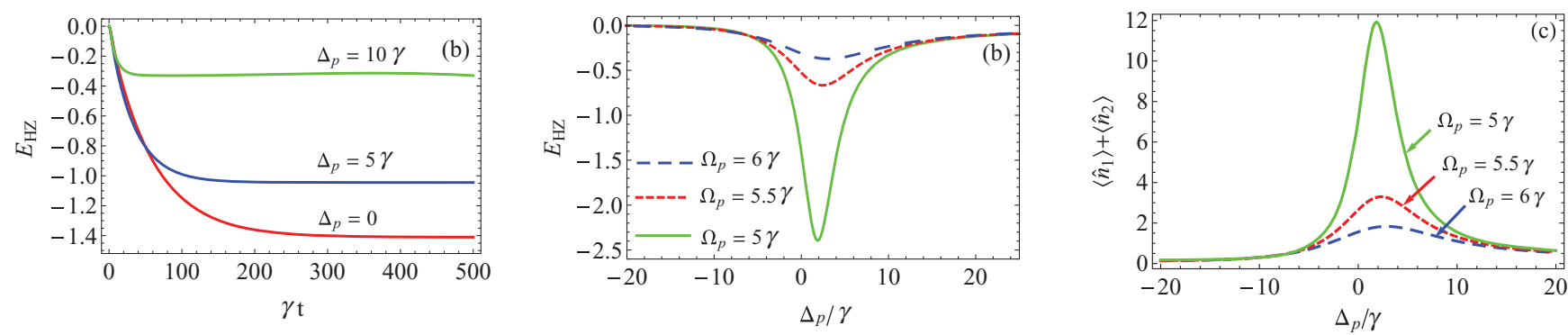

FIG. 4. (Color online) Plots of $E_{\mathrm{HZ}}$ versus $\gamma t$ for $\Omega_{p}=5 \gamma$ for various values of $\Delta_{p}$ and with cavity modes initially in a vacuum state. (b) Plots of $E_{\mathrm{HZ}}$ in the steady state versus $\Delta_{p}$ for various values of the Rabi frequency of the pump field $\Omega_{p}$. (c) Total mean photon number versus $\Delta_{p}$ for various values of $\Omega_{p}$. All other parameters are the same as in Fig. 2.

long-time limit, indicating the existence of steady state squeezing. In this respect, we study the dependence of the steady state two-mode squeezing on detuning and amplitude of the pump laser field. As shown in Fig. 3(c), the squeezing crucially depends on both the detuning $\Delta_{p}$ and Rabi frequency $\Omega_{p}$ of the pump laser. We observe that optimum squeezing appears at zero detuning for $\Omega_{p}=5 \gamma$-the minimum value of the Rabi frequency for which a steady state solution exists. When the Rabi frequency increases beyond this value, the value of the detunings that give optimum squeezing slightly shift to the right (positive detuning). The other key aspect is that, when the Rabi frequency increases, the range of detuning for which the squeezing occurs becomes wider while the maximum squeezing decreases.

\section{Entanglement of cavity modes}

Here we present the transient and steady state behavior of the entanglement as a function of laser detuning and strength of coherent field. It is worthwhile to mention here that, since our scheme involves two-photon emissions and annihilations as ascribed by the master equation, the type of entanglement we expect is of the form $\left|1_{1} 1_{2}\right\rangle \pm\left|0_{1} 0_{2}\right\rangle$. Not all two-mode entanglement criteria can detect such type of entanglement. The class of inequalities that can detect this entanglement include a particular Hillery-Zubairy [22] criterion, logarithmic negativity [23-25], and Duan-Giedke-Cirac-Zoller (DGCZ) [26] criterion which all depend on second-order moments. Here we employ all the three inseparability criteria above to study the entanglement properties of the cavity radiation. In particular, we show that, for certain combinations of the quadrature operators, the DGCZ criterion reduces to the two-mode squeezing condition. Note also that, since the cavity modes do not exhibit Gaussian statistics, these tests are only sufficient to determine whether the cavity field is in an entangled state or not.

\section{Hillery-Zubairy criterion}

The Hillery-Zubairy (HZ) criterion, which depends on second-order moments of the cavity-mode operators, states that the state of a two-mode system is said to be entangled if the following inequality is satisfied:

$$
E_{\mathrm{HZ}} \equiv\left\langle n_{1}\right\rangle\left\langle n_{2}\right\rangle-\left|\left\langle a_{1} a_{2}\right\rangle\right|^{2}<0 ;
$$

that is, for the cavity modes to be entangled, the product of the mean photon number in each mode should be smaller than the modulus squared of the intermode correlation. In Fig. 4(a), we plot $E_{\mathrm{HZ}}$ versus dimensionless time $\gamma t$ for various values of detuning $\Delta_{p}$. We assume that the cavity modes are initially in a vacuum state and the atom is prepared in a coherent superposition of the levels $|a\rangle$ and $|b\rangle$. We see from this figure that the two cavity modes are entangled at all times for the given parameters. The function $E_{\mathrm{HZ}}$ approaches to zero (no entanglement) as the pump laser detuning increases. Recall that the strength the intermode correlation becomes weaker when the pump laser detuning increases. Since the source of entanglement is the correlation between the photons, it is anticipated to see weaker entanglement at large detunings.

Figure 4(b) shows the dependence of the steady state entanglement on the pump laser detuning and Rabi frequency $\Omega_{p}$. This figure shows that the function $E_{\mathrm{HZ}}$ has a dip (negative value) in the vicinity of resonance $\left(\Delta_{p}=0\right)$. The center of the dip slightly shifts to the right when the Rabi frequency is increased; the dips are centered at $\Delta_{p} / \gamma=$ $1.92,2.52$, and 3.12 corresponding to $\Omega_{p}=5.0 \gamma, 5.5 \gamma$, and $6.0 \gamma$, respectively. For larger detunings, the function $E_{\mathrm{HZ}}$ approaches zero, indicating disentanglement of the cavity modes. Furthermore, to gain insight into the relation between entanglement and number of photons in the cavity, we show in Fig. 4(c) the total mean photon number of the cavity field as a function of detuning and pump Rabi frequency for the same parameters as in Fig. 4(b). The intensity of the cavity field increases with decreasing Rabi frequency of the pump field. This is encouraging because the emitted light is relatively intense when in the regime where the entanglement is robust, making our scheme a source of bright entangled light. Another interesting aspect is that, although one would naively expect the maximum intensity to occur exactly at resonance, it rather appears at a small positive detuning similar to Fig. 4(b).

\section{Logarithmic negativity}

The entanglement generated can also be determined by applying logarithmic negativity, which is necessary and sufficient entanglement monotone for Gaussian states and only sufficient for non-Gaussian states [23-25]. Recently, the method of logarithmic negativity has been used to test continuous-variable entanglement in a three-level cascade laser with a parametric oscillator [31]. It has been shown that logarithmic negativity can be used as an alternative approach to 
test entanglement in two-mode continuous-variables systems. The logarithmic negativity for a two-mode state is defined as

$$
E_{N}=\max \left[0,-\log _{2} \mathcal{V}\right],
$$

where $\mathcal{V}$ is the smallest eigenvalue of the simplistic matrix which is given by [25]

$$
\mathcal{V}=\frac{1}{\sqrt{2}}\left[\sigma+\sqrt{\sigma^{2}-4 \operatorname{det} \Upsilon}\right]^{1 / 2},
$$

with $\sigma=\operatorname{det} \mathcal{A}_{1}+\operatorname{det} \mathcal{A}_{2}-2 \operatorname{det} \mathcal{A}_{12}$. Here, $\mathcal{A}_{1}, \mathcal{A}_{2}$, and $\mathcal{A}_{12}$ are $2 \times 2$ matrices that appear in the covariant matrix

$$
\Upsilon=\left(\begin{array}{ll}
\mathcal{A}_{1} & \mathcal{A}_{12} \\
\mathcal{A}_{12}^{T} & \mathcal{A}_{2}
\end{array}\right) .
$$

The elements of the covariant matrix are given by

$$
\Upsilon_{i j}=\frac{1}{2}\left\langle\hat{X}_{i} \hat{X}_{j}+\hat{X}_{j} \hat{X}_{i}\right\rangle-\left\langle\hat{X}_{i}\right\rangle\left\langle\hat{X}_{j}\right\rangle
$$

where the quadrature operators for each mode are defined as $\hat{X}_{1}=\hat{a}_{1}^{\dagger}+\hat{a}_{1}, \hat{X}_{2}=i\left(\hat{a}_{1}^{\dagger}-\hat{a}_{1}\right), \hat{X}_{3}=\hat{a}_{2}^{\dagger}+\hat{a}_{2}$, and $\hat{X}_{4}=$ $i\left(\hat{a}_{2}^{\dagger}-\hat{a}_{2}\right)$. For the cavity modes initially in a vacuum state, the covariant matrix has the simple form

$$
\Upsilon=\left(\begin{array}{cccc}
m & 0 & c & 0 \\
0 & m & 0 & -c \\
c & 0 & n & 0 \\
0 & -c & 0 & n
\end{array}\right),
$$

where $m=\left\langle\hat{a}_{1}^{\dagger} \hat{a}_{1}\right\rangle+\left\langle\hat{a}_{1} \hat{a}_{1}^{\dagger}\right\rangle, n=\left\langle\hat{a}_{2}^{\dagger} \hat{a}_{2}\right\rangle+\left\langle\hat{a}_{2} \hat{a}_{2}^{\dagger}\right\rangle$, and $c=$ $\left\langle\hat{a}_{1} \hat{a}_{2}\right\rangle+\left\langle\hat{a}_{1}^{\dagger} \hat{a}_{2}^{\dagger}\right\rangle$. Based on this criteria, the two modes are said to be entangled when the logarithmic negativity $E_{N}$ is positive which, according to (8), implies that $\log _{2} \mathcal{V}$ should be negative or $\mathcal{V}<1$. Thus one might consider $\mathcal{V}<1$ as a sufficient condition to detect entanglement in our system.

Figure 5(a) shows the smallest eigenvalue $\mathcal{V}$ in the steady state as a function of the Rabi frequency of the pump field. It is clear from this figure that the cavity radiation exhibits entanglement, which gradually dies out when the detuning increases. The smallest eigenvalue strongly relies on the strength of the pump laser. As shown in Fig. 5(a), the width of the dip of the smallest eigenvalue becomes larger as the Rabi frequency $\Omega_{p}$ of the pump laser increases. The minimum of the dip occurs for small Rabi frequency, showing better entanglement. Ironically, the minimum value of $\mathcal{V}$ does not occur at resonance and shifts away to the right for larger
Rabi frequency. While the minimum value for $\mathcal{V}$ decreases with increasing $\Omega_{p}$, the interval of detuning for which the entanglement exists become wider for larger Rabi frequency.

\section{DGCZ criterion}

To make a connection between the two-mode squeezing and entanglement, we next consider the criterion that is based on the quadrature of the cavity field [26]. According to DGCZ, the state of a system is known to be entangled if the quantum fluctuations of the two Einstein-Podolsky-Rosen-like operator $\hat{u}$ and $\hat{v}$ of the two modes satisfy the inequality

$$
D=\Delta u^{2}+\Delta v^{2}<2,
$$

where we define $\hat{u}=\hat{X}_{a_{1}}-\hat{X}_{a_{2}}$ and $\hat{v}=\hat{P}_{a_{1}}+\hat{P}_{a_{2}}$ differently with $\hat{X}_{j}=\left(\hat{\jmath}^{\dagger}+\hat{\jmath}\right) / \sqrt{2}, \hat{P}_{j}=i\left(\hat{\jmath}^{\dagger}-\hat{\jmath}\right) / \sqrt{2}$ (with $\left.\hat{\jmath}=\hat{a}_{1}, \hat{a}_{2}\right)$ being the quadrature operators for the two modes of the cavity field. It is not difficult to show that, for the cavity modes initially in a vacuum state, the sum of the variances (13) has a form

$$
D=2\left(1+\left\langle\hat{n}_{1}\right\rangle+\left\langle\hat{n}_{2}\right\rangle-2 \operatorname{Re}\left\langle\hat{a}_{1} \hat{a}_{2}\right\rangle\right)=2 \Delta c_{-}^{2} .
$$

This is a direct relationship between two-mode squeezing and entanglement of the cavity field. Similarly, if we had defined $\hat{u}=\hat{X}_{a_{1}}+\hat{X}_{a_{2}}$ and $\hat{v}=\hat{P}_{a_{1}}-\hat{P}_{a_{2}}$ we would have had $\Delta u^{2}+$ $\Delta v^{2}=2 \Delta c_{+}^{2}$. Therefore, whenever there is squeezing in the system the cavity field exhibits entanglement.

In order to gain insight into the connection between the inseparability criteria used to detect entanglement in our system, we illustrate all three criteria in Fig. 5 for the same set of parameters. Although the three criteria show qualitatively similar behavior as a function of pump laser detuning and Rabi frequency, there is notable disparity among the entanglement detected. For example, for $\Omega_{p}=10 \gamma$, the logarithmic negativity and DGCZ criteria exhibit entanglement for a certain range of detuning., with the logarithmic negativity showing entanglement for relatively wider detuning. However, the HZ criteria shows entanglement for a wider range, mostly in the positive detuning territory. When one increases the Rabi frequency further, all three criteria show similar behavior. Since there is no physical reason that the entanglement be "switched on" or "switched off" at a particular detuning, as indicated in the logarithmic negativity and DGCZ criteria, we thus note that the $\mathrm{HZ}$ is a stronger criterion than DGCZ to detect entanglement in our scheme. It is worth noting that the logarithmic negativity and DGCZ criteria are
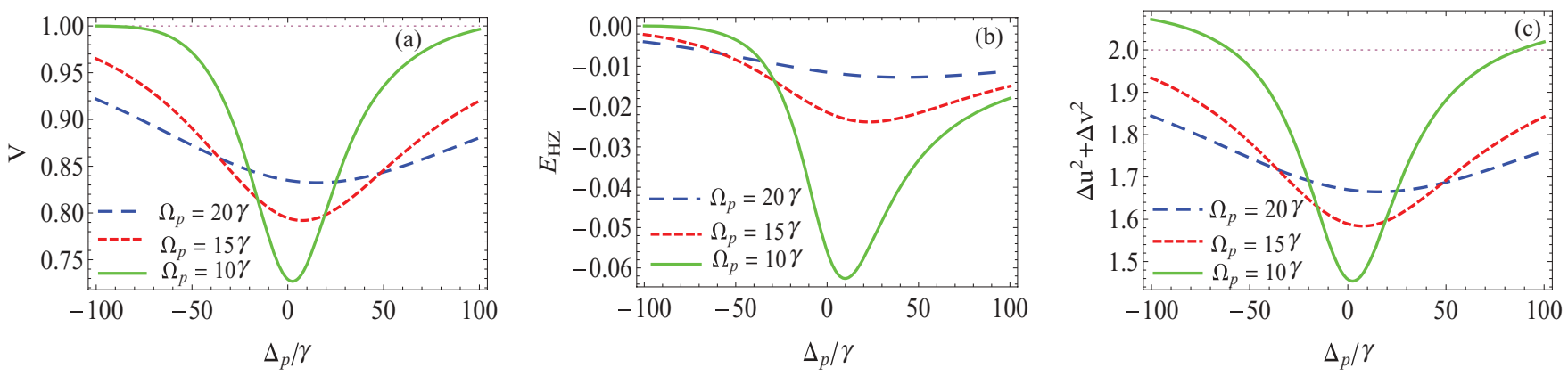

FIG. 5. (Color online) Plots of entanglement criteria: (a) smallest eigenvalue $\mathcal{V}$, (b) HZ ( $\left.E_{\mathrm{HZ}}\right)$, and (c) DGCZ $\left(\Delta u^{2}+\Delta v^{2}\right)$ versus $\Delta_{p} / \gamma$ for various values of $\Omega_{p}$. All other parameters and initial conditions are the same as in Fig. 2. 
based on quadrature operators of the cavity modes and hence have similar behavior, as illustrated in Figs. 5(a) and 5(c). Conversely, HZ depends on the photon number and intermodal correlations. The $\mathrm{HZ}$ criteria depends on the magnitude of the intermode correlation while the other criteria (squeezing, $E_{N}$ and DGCZ) depend on its magnitude as well as its phase.

\section{CONCLUSION}

We have shown that the cavity field of a single-atom double Raman laser can exhibit two-mode squeezing and entanglement both in transient and steady state regimes. Atomic spontaneous emission and cavity losses have been taken into account in our analysis. We found that the existence of squeezing in the system crucially relies on the intermode correlation measured via $\left\langle\hat{a}_{1} \hat{a}_{2}\right\rangle$. Whenever this correlation exceeds the total mean photon number of the cavity field, which requires tuning several system parameters, the cavity field starts to exhibit squeezing. This transient squeezing survives for a long time and reaches the steady state regime. In both transient and steady state cases, the squeezing depends on detuning and amplitude of the pump laser. The higher amplitude and detuning, the lower the squeezing becomes. Based on one of the criteria we used, the entanglement of the cavity field also depends on the intermode correlation, but this time, the modulus of the correlation has to be larger than the product of the mean photon number in each mode.
Our results also show that the cavity field exhibits transient as well as steady state entanglement for a wide range of control parameters. Moreover, we have established a direct relationship between two-mode squeezing and entanglement. This link is particularly not surprising given that both originate from the quantum coherence induced by the driving laser fields. The fact that the entanglement generated is detected by three different entanglement criteria makes our scheme a viable candidate for a source of robust two-mode continuousvariable entanglement.

\section{ACKNOWLEDGMENTS}

We thank M. S. Zubairy for useful discussions on entanglement criteria. E.A.S. is supported by the Herman F. Heep and Minnie Belle Heep Texas A\&M University Endowed Fund held and administered by the Texas A\&M Foundation. R.O. is supported by the Ministry of Higher Education (MOHE)/University of Malaya HIR Grant No. A-00000450001 and the MOHE ERGS Grant No. ER014-2011A.

\section{APPENDIX: EQUATIONS FOR EXPECTATION VALUES OF CAVITY-MODE OPERATORS}

Using the relation $d\langle a\rangle / d t=\operatorname{Tr}[(d \rho / d t) a]$ together with the master equation, we obtain the time evolution of the expectation values of the cavity-mode operators to be

$$
\begin{aligned}
\frac{d}{d t}\left\langle\hat{a}_{1}\right\rangle & =-\left(\mathcal{L}_{1}^{*}-\mathcal{G}_{1}^{*}\right)\left\langle\hat{a}_{1}\right\rangle+e^{i \varphi_{t}}\left(\mathcal{Q}_{1}^{*}-\mathcal{Q}_{3}^{*}\right)\left\langle\hat{a}_{2}^{\dagger}\right\rangle, \\
\frac{d}{d t}\left\langle\hat{a}_{2}\right\rangle & =-\left(\mathcal{L}_{2}^{*}-\mathcal{G}_{2}^{*}\right)\left\langle\hat{a}_{2}\right\rangle+e^{i \varphi_{t}}\left(\mathcal{Q}_{2}^{*}-\mathcal{Q}_{3}^{*}\right)\left\langle\hat{a}_{1}^{\dagger}\right\rangle, \\
\frac{d}{d t}\left\langle\hat{a}_{1}^{\dagger}\right\rangle & =-2\left(\mathcal{L}_{1}-\mathcal{G}_{1}\right)\left\langle\hat{a}_{1}^{\dagger}\right\rangle+2 e^{-i \varphi_{t}}\left(\mathcal{Q}_{1}-\mathcal{Q}_{3}\right)\left\langle\hat{a}_{1}^{\dagger} \hat{a}_{2}\right\rangle, \\
\frac{d}{d t}\left\langle\hat{a}_{2}^{2}\right\rangle & =-2\left(\mathcal{L}_{2}^{*}-\mathcal{G}_{2}^{*}\right)\left\langle\hat{a}_{2}^{2}\right\rangle+2 e^{i \varphi_{t}}\left(\mathcal{Q}_{2}^{*}-\mathcal{Q}_{3}^{*}\right)\left\langle\hat{a}_{1}^{\dagger} \hat{a}_{2}\right\rangle, \\
\frac{d}{d t}\left\langle\hat{a}_{1}^{\dagger} \hat{a}_{2}\right\rangle & =-\left(\mathcal{L}_{1}-\mathcal{G}_{1}+\mathcal{L}_{2}^{*}-\mathcal{G}_{2}^{*}\right)\left\langle\hat{a}_{1}^{\dagger} \hat{a}_{2}\right\rangle+e^{i \varphi_{t}}\left(\mathcal{Q}_{2}^{*}-\mathcal{Q}_{3}^{*}\right)\left\langle\hat{a}_{1}^{\dagger}\right\rangle+e^{-i \varphi_{t}}\left(\mathcal{Q}_{1}-\mathcal{Q}_{3}\right)\left\langle\hat{a}_{2}^{2}\right\rangle, \\
\frac{d}{d t}\left\langle\hat{a}_{1} \hat{a}_{2}\right\rangle & =-\left(\mathcal{L}_{1}^{*}+\mathcal{L}_{2}^{*}-\mathcal{G}_{1}^{*}-\mathcal{G}_{2}^{*}\right)\left\langle\hat{a}_{1} \hat{a}_{2}\right\rangle+e^{i \varphi_{t}}\left[\left(\mathcal{Q}_{1}^{*}-\mathcal{Q}_{3}^{*}\right)\left\langle\hat{a}_{2}^{\dagger} \hat{a}_{2}\right\rangle+\left(\mathcal{Q}_{2}^{*}-\mathcal{Q}_{3}^{*}\right)\left\langle\hat{a}_{1}^{\dagger} \hat{a}_{1}\right\rangle-\mathcal{Q}_{3}^{*}\right], \\
\frac{d}{d t}\left\langle n_{1}\right\rangle & =-\left(\mathcal{L}_{1}+\mathcal{L}_{1}^{*}-\mathcal{G}_{1}-\mathcal{G}_{1}^{*}\right)\left\langle\hat{a}_{1}^{\dagger} \hat{a}_{1}\right\rangle+e^{i \varphi_{t}}\left(\mathcal{Q}_{1}^{*}-\mathcal{Q}_{3}^{*}\right)\left\langle\hat{a}_{1}^{\dagger} \hat{a}_{2}^{\dagger}\right\rangle+e^{-i \varphi_{t}}\left(\mathcal{Q}_{1}-\mathcal{Q}_{3}\right)\left\langle\hat{a}_{1} \hat{a}_{2}\right\rangle+\mathcal{G}_{1}+\mathcal{G}_{1}^{*}, \\
\frac{d}{d t}\left\langle n_{2}\right\rangle & =-\left(\mathcal{L}_{2}+\mathcal{L}_{2}^{*}-\mathcal{G}_{2}-\mathcal{G}_{2}^{*}\right)\left\langle\hat{a}_{1}^{\dagger} \hat{a}_{1}\right\rangle+e^{i \varphi_{t}}\left(\mathcal{Q}_{2}^{*}-\mathcal{Q}_{3}^{*}\right)\left\langle\hat{a}_{1}^{\dagger} \hat{a}_{2}^{\dagger}\right\rangle+e^{-i \varphi_{t}}\left(\mathcal{Q}_{2}-\mathcal{Q}_{3}\right)\left\langle\hat{a}_{1} \hat{a}_{2}\right\rangle+\mathcal{G}_{2}+\mathcal{G}_{2}^{*},
\end{aligned}
$$

where

$$
\begin{aligned}
& \mathcal{L}_{1}=\frac{\left|\mathrm{g}_{1}\right|^{2}}{\chi}\left[-i \Omega_{c}\left(\Omega_{p}^{2}-\Omega_{c}^{2}-\Gamma_{a c}^{*} \Gamma_{b c}^{*}\right) \rho_{a b}^{(0)}+\left(\Gamma_{a c}^{*} \Gamma_{a d}^{*} \Gamma_{b c}^{*}+\Omega_{p}^{2} \Gamma_{b c}^{*}+\Omega_{c}^{2} \Gamma_{a d}^{*}\right) \rho_{b b}^{(0)}\right]+\kappa_{1}, \\
& \mathcal{G}_{1}=\frac{\left|\mathrm{g}_{1}\right|^{2}}{\chi}\left[-i \Omega_{p}\left(\Omega_{p}^{2}-\Omega_{c}^{2}+\Gamma_{a c}^{*} \Gamma_{a d}^{*}\right) \rho_{d c}^{(0)}+\left(\Gamma_{a c}^{*} \Gamma_{a d}^{*} \Gamma_{b c}^{*}+\Omega_{p}^{2} \Gamma_{b c}^{*}+\Omega_{c}^{2} \Gamma_{a d}^{*}\right) \rho_{d d}^{(0)}\right], \\
& \mathcal{L}_{2}=\frac{\left|\mathrm{g}_{2}\right|^{2}}{\chi}\left[-i \Omega_{p}\left(\Omega_{p}^{2}-\Omega_{c}^{2}+\Gamma_{b c}^{*} \Gamma_{d b}^{*}\right) \rho_{c d}^{(0)}+\left(\Gamma_{a d}^{*} \Gamma_{b c}^{*} \Gamma_{d b}+\Omega_{p}^{2} \Gamma_{a d}^{*}+\Omega_{c}^{2} \Gamma_{b c}^{*}\right) \rho_{c c}^{(0)}\right]+\kappa_{2}, \\
& \mathcal{G}_{2}=\frac{\left|\mathrm{g}_{2}\right|^{2}}{\chi}\left[-i \Omega_{c}\left(\Omega_{p}^{2}-\Omega_{c}^{2}-\Gamma_{a d}^{*} \Gamma_{d b}\right) \rho_{b a}^{(0)}+\left(\Gamma_{a d}^{*} \Gamma_{b c}^{*} \Gamma_{d b}+\Omega_{p}^{2} \Gamma_{a d}^{*}+\Omega_{c}^{2} \Gamma_{b c}^{*}\right) \rho_{a a}^{(0)}\right], \\
& \mathcal{Q}_{1}=\frac{\mathrm{g}_{1} \mathrm{~g}_{2}}{\chi}\left[\Omega_{p} \Omega_{c}\left(\Gamma_{b c}^{*}+\Gamma_{a d}^{*}\right) \rho_{c c}^{(0)}+\Omega_{c} \Omega_{p}\left(\Gamma_{b c}+\Gamma_{a d}\right) \rho_{d d}^{(0)}+i \Omega_{c}\left(\Gamma_{a c}^{*} \Gamma_{b c}^{*}-\Gamma_{a d} \Gamma_{d b}^{*}\right) \rho_{c d}^{(0)}\right],
\end{aligned}
$$




$$
\begin{aligned}
& \mathcal{Q}_{2}=\frac{\mathrm{g}_{1} \mathrm{~g}_{2}}{\chi}\left[\Omega_{p} \Omega_{c}\left(\Gamma_{b c}^{*}+\Gamma_{a d}^{*}\right) \rho_{a a}^{(0)}+\Omega_{c} \Omega_{p}\left(\Gamma_{b c}+\Gamma_{a d}\right) \rho_{b b}^{(0)}+i \Omega_{p}\left(\Gamma_{b c} \Gamma_{d b}^{*}-\Gamma_{a d} \Gamma_{a c}^{*}\right) \rho_{b a}^{(0)}\right], \\
& \mathcal{Q}_{3}=\frac{\mathrm{g}_{1} \mathrm{~g}_{2}}{\chi}\left[\Omega_{p} \Omega_{c}\left(\Gamma_{b c}^{*}+\Gamma_{a d}^{*}\right) \rho_{a a}^{(0)}+\Omega_{c} \Omega_{p}\left(\Gamma_{b c}+\Gamma_{a d}\right) \rho_{d d}^{(0)}+i \Omega_{p}\left(\Omega_{p}^{2}-\Omega_{c}^{2}+\Gamma_{a c}^{*} \Gamma_{a d}^{*}\right) \rho_{b a}^{(0)}+i \Omega_{c}\left(\Omega_{p}^{2}-\Omega_{c}^{2}-\Gamma_{a d} \Gamma_{d b}^{*}\right) \rho_{c d}^{(0)}\right], \\
& \mathcal{Q}_{4}=\frac{\mathrm{g}_{1} \mathrm{~g}_{2}}{\chi}\left[\Omega_{p} \Omega_{c}\left(\Gamma_{b c}^{*}+\Gamma_{a d}^{*}\right) \rho_{c c}^{(0)}+\Omega_{c} \Omega_{p}\left(\Gamma_{b c}+\Gamma_{a d}\right) \rho_{b b}^{(0)}+i \Omega_{p}\left(\Omega_{p}^{2}-\Omega_{c}^{2}+\Gamma_{b c} \Gamma_{d b}^{*}\right) \rho_{b a}^{(0)}-i \Omega_{c}\left(\Omega_{p}^{2}-\Omega_{c}^{2}-\Gamma_{a c}^{*} \Gamma_{b c}^{*}\right) \rho_{c d}^{(0)}\right],
\end{aligned}
$$

with

$$
\chi=\Gamma_{a c}^{*} \Gamma_{a d}^{*} \Gamma_{b c}^{*} \Gamma_{d b}+\Omega_{p}^{2}\left(\Gamma_{a c}^{*} \Gamma_{a d}^{*}+\Gamma_{b c}^{*} \Gamma_{d b}\right)+\Omega_{c}^{2}\left(\Gamma_{a c}^{*} \Gamma_{b c}^{*}+\Gamma_{a d}^{*} \Gamma_{d b}\right)+\left(\Omega_{p}-\Omega_{c}\right)^{2},
$$

with $\rho_{i i}^{(0)}$ and $\rho_{i j}^{(0)}(i, j=a, b, c, d)$ being initial populations and coherences, respectively. $\Gamma_{a c}=i \Delta_{2}+\gamma_{a c}, \Gamma_{a d}=i\left(\Delta_{c}-\Delta_{1}\right)+\gamma_{a d}$, $\Gamma_{b c}=i\left(\Delta_{p}-\Delta_{1}\right)+\gamma_{b c}$, and $\Gamma_{d b}=i \Delta_{1}+\gamma_{d b}$ are complex dephasings.

[1] C. M. Caves, Phys. Rev. D 26, 1817 (1982).

[2] D. F. Walls, Nature (London) 306, 141 (1983).

[3] A. Heidmann, R. J. Horowicz, S. Reynaud, E. Giacobino, C. Fabre, and G. Camy, Phys. Rev. Lett. 59, 2555 (1987).

[4] R. E. Slusher, L. W. Hollberg, B. Yurke, J. C. Mertz, and J. F. Valley, Phys. Rev. Lett. 55, 2409 (1985).

[5] S. Machida, Y. Yamamoto, and Y. Itaya, Phys. Rev. Lett. 58, 1000 (1987).

[6] C. M. Caves, Phys. Rev. D 23, 1693 (1981).

[7] Y. Yamamoto and H. A. Haus, Rev. Mod. Phys. 58, 1001 (1986).

[8] C. H. Bennett, G. Brassard, C. Crepeau, R. Jozsa, A. Peres, and W. K. Wootters, Phys. Rev. Lett. 70, 1895 (1993).

[9] D. Bouwmeester, J. W. Pan, K. Mattle, M. Eibl, H. Weinfurter, and A. Zeilinger, Nature (London) 390, 575 (1997).

[10] C. H. Bennett and G. Brassard, in Proceedings of the IEEE International Conference on Computers, Systems and Signal Processing (IEEE Computer Society Press, Los Alamitos, 1984), p. 175.

[11] S. L. Braunstein and P. van Look, Rev. Mod. Phys. 77, 513 (2005).

[12] H. Xiong, M. O. Scully, and M. S. Zubairy, Phys. Rev. Lett. 94, 023601 (2005).

[13] E. Alebachew, Phys. Rev. A 76, 023808 (2007).

[14] E. A. Sete, Opt. Commun. 281, 6124 (2008).

[15] E. Alebachew, Opt. Commun. 280, 133 (2007).

[16] C. H. Raymond Ooi, Phys. Rev. A 76, 013809 (2007).

[17] S. Qamar, F. Ghafoor, M. Hillery, and M. S. Zubairy, Phys. Rev. A 77, 062308 (2008).
[18] A. P. Fang, Y. L. Chen, F. L. Li, H. R. Li, and P. Zhang, Phys. Rev. A 81, 012323 (2010).

[19] R. Tahira, M. Ikram, H. Nha, and M. S. Zubairy, Phys. Rev. A 83, 054304 (2011); Eyob A. Sete, ibid. 84, 063808 (2011); J. Phys. B: At., Mol. Opt. Phys. 44, 225504 (2011).

[20] M. Kiffner, M. S. Zubairy, J. Evers, and C. H. Keitel, Phys. Rev. A 75, 033816 (2007).

[21] J. McKeever, A. Boca, A. D. Boozer, J. R. Buck, and H. J. Kimble, Nature (London) 425, 268 (2003).

[22] M. Hillery and M. S. Zubairy, Phys. Rev. Lett. 96, 050503 (2006).

[23] G. Vidal and R. F. Werner, Phys. Rev. A 65, 032314 (2002).

[24] J. Laurat, G. Keller, J. O. H. Augusto, C. Fabre, T. Coudreau, A. Serafim, G. Adesso, and F. Illuminati, J. Opt. B 7, 577 (2005).

[25] G. Adesso, A. Serafini, and F. Illuminati, Phys. Rev. A 70, 022318 (2004).

[26] L. M. Duan, G. Giedke, J. I. Cirac, and P. Zoller, Phys. Rev. Lett. 84, 2722 (2000).

[27] G. S. Agarwal and A. Biswas, New J. Phys. 7, 211 (2005).

[28] H. Nha and J. Kim, Phys. Rev. A 74, 012317 (2006).

[29] M. O. Scully and M. S. Zubairy, Quantum Optics (Cambridge University Press, Cambridge, 1997).

[30] M. P. Yuen and J. H. Shapiro, IEEE Trans. Inf. Theory 26, 78 (1980).

[31] Y.-H. Ma, Q.-X. Mu, G.-H. Yang, and L. Zhou, J. Phys. B: At., Mol. Opt. Phys. 41, 215502 (2008). 